\title{
Analysis of Transient Stability for Multimachine Power System
}

\author{
${ }^{1}$ Abhishek Verma, ${ }^{2}$ Ashutosh Shukla, ${ }^{3}$ Ashwani Kumar, ${ }^{4}$ Amit Kumar \\ Department of Electrical and Electronics Engineering, College of Engineering Roorkee, Roorkee, \\ India. abhishek.vermaee3@gmail.com, ashutiet@gmail.com, ashwani.ee15@gmail.com, \\ amitkee05@gmail.com
}

\begin{abstract}
The stability is an important guarantee for the safe and reliable operation of the power system, it is a decisive factor to limit the transmission distance and conveying capacity of the power system. Therefore, various measures must be taken to improve the system stability. The stability of the power system includes power angle stability, voltage stability and frequency stability. Among them, the power angle stability includes static stability and transient stability. Considering the importance of stability to the power system, the power system stability analysis methods and the measures to improve the power system stability is studied, so as to provide some useful reference for the safe and reliable operation of the power system. Generally, for multi-machine system transient stability becomes a major concern of wide power system due to load demand increasing day by day. In order to withstand disturbances with power quality issues requires evaluation of power system's ability. There are many different kinds of methods for analysis of transient stability usually for multi-machine system. These methods include extended equal area criteria, time domain analysis and direct stability methods such as the transient energy function.
\end{abstract}

Keywords - Transient Stability, Power System, Multi-machine.

\section{INTRODUCTION}

The power system will be interfered by many factors, the vast majority of which are small signal interferences. We can obtain the system unstable way by establishing an appropriate small signal stability analysis model and analysing the stability of the power system, and then we can study the preventive measures of instability to improve system stability. Therefore, the small signal stability analysis of the power system is of great significance, this paper will study two commonly used methods of small signal stability analysis. The classifications of Power System Stability is shown in Fig. 1.

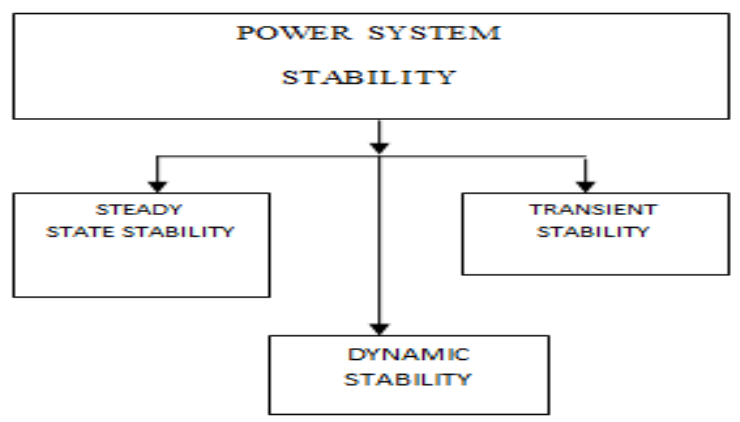

Fig. 1: Block Diagram for Types of Stability stability of the power systems, where some of them have directly focused on the transient stability, in-depth. From a physical point of view, transient stability can be defined as the ability of a system to remain with synchronous outcomes during the occurrence of large perturbations. Severe type of disturbance is a short circuit. When a fault occurs, the electrical powers from a generators located in remote areas affected more than the generators located in nearby. It has been observed that some of the electrical power system may be stable even after the fault and some of them becomes stable after clearing the fault with sufficient rapidity. Transient stability limit depends on the type of location, disturbance and magnitude of disturbance.

\section{ILLUSTRATIVE SYSTEM EXAMPLE}

We have worked on 3-machine, 9-bus system shown in Fig. 2.

The transient stability is for effectively influenced as compare with steady state on power system. A large number of investigations are dedicated to the types of 


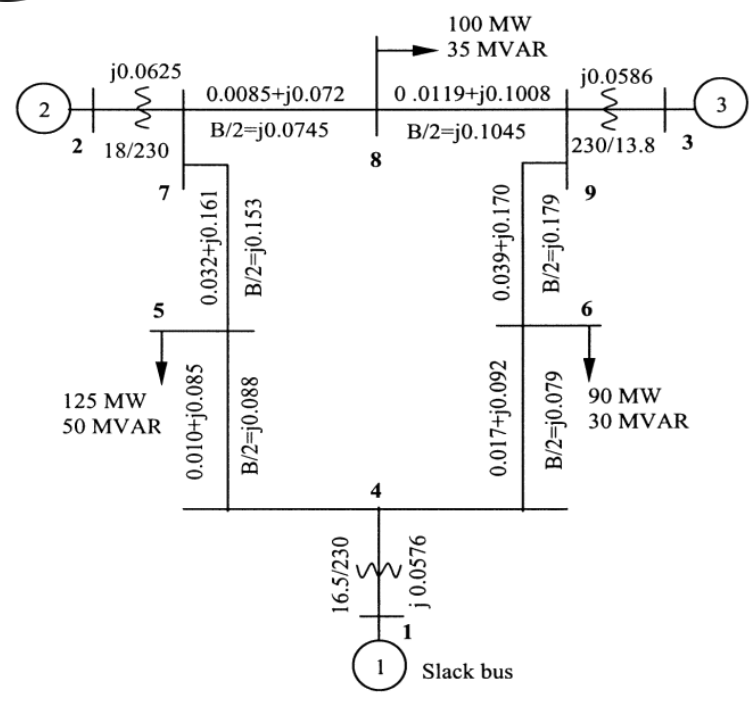

Fig. 2: 3-machine, 9-bus system; impedances are in per unit on base of $100 \mathrm{MVA}$

\section{MATHEMATICAL MODELLING}

Y-matrix for each electrical network condition (pre-fault, during and after fault) is solved once then we can remove all nodes except for internal generator nodes and we will get the Y-matrix for reduced network. Remember the reduced network matrix can be obtained by matrix operation by consider all nodes have zero injection currents except for internal generator nodes. With ' $n$ ' number of generators in a power system network, the nodal equation can be written as: $\left[\begin{array}{c}\mathrm{I}_{\mathrm{n}} \\ 0\end{array}\right]=\left[\begin{array}{ll}\mathrm{Y}_{\mathrm{nn}} & \mathrm{Y}_{\mathrm{nr}} \\ \mathrm{Y}_{\mathrm{rn}} & \mathrm{Y}_{\mathrm{rr}}\end{array}\right]\left[\begin{array}{c}\mathrm{V}_{\mathrm{n}} \\ \mathrm{V}_{\mathrm{r}}\end{array}\right]$

we substitute $\mathrm{V}_{\mathrm{r}}$ from here and to find

$\mathrm{I}_{\mathrm{n}}=\left(\mathrm{Y}_{\mathrm{nn}}-\mathrm{Y}_{\mathrm{nr}} \mathrm{Y}_{\mathrm{rr}}^{-1} \mathrm{Y}_{\mathrm{rn}}\right) \mathrm{V}_{\mathrm{n}}$

We will get the required reduced matrix which can be written as follows:

$\mathrm{Y}_{\mathrm{R}}=\mathrm{Y}_{\mathrm{nn}}-\mathrm{Y}_{\mathrm{nr}} \mathrm{Y}_{\mathrm{rr}}^{-1} \mathrm{Y}_{\mathrm{rn}}$

As it has ' $n$ ' number of generators with dimensions $(n \times$ $n)$. The analytical technique can be used only for the loads acted as constant impedances as shown by equations (1)-
(3). The reduced matrices are calculated for the power system stability. The resultant matrices before, during and after fault is given in Appendix II. At node ' $i$ ' the electrical power output of machine is given by:

$P_{e i}=E_{i}^{2} G_{i i}+\sum_{j=1}^{n} E_{i} E_{j} E_{i j} \cos \left(\theta_{i j}-\delta_{i}+\delta_{j}\right) \quad i=$

$1,2,3 \ldots \ldots \mathrm{n}$

$\bar{Y}_{i j}=Y_{i j} \angle \theta_{i j}=G_{i j}+j B_{i j}$

$\bar{\Upsilon}_{\mathrm{ii}}=\mathrm{Y}_{\mathrm{ii}} \angle \theta_{\mathrm{i}}=\mathrm{G}_{\mathrm{ii}}+\mathrm{j} \mathrm{B}_{\mathrm{ii}}$

The mechanical power equations are then given by:

$\frac{2 H_{i}}{\omega_{R}} \frac{d \omega_{j}}{d t}+D_{i} \omega_{j}=P_{m i}-\left[E_{i}^{2} G_{i i}+\sum_{j=1}^{n} E_{i} E_{j} E_{i j} \cos \left(\theta_{i j}-\right.\right.$ $\left.\left.\delta_{\mathrm{i}}+\delta_{\mathrm{j}}\right)\right] \quad(7) \quad$ It must be observed that before the disturbance at $\mathrm{t}=0 ; \mathrm{P}_{\text {mio }}=\mathrm{P}_{\text {eio }}$; Thereby,

$\mathrm{P}_{\text {mio }}=\mathrm{E}_{\mathrm{i}}^{2} \mathrm{G}_{\mathrm{iio}}+\sum_{\mathrm{j}=1}^{\mathrm{n}} \mathrm{E}_{\mathrm{i}} \mathrm{E}_{\mathrm{j}} \mathrm{E}_{\mathrm{ijo}} \cos \left(\theta_{\mathrm{ijo}}-\delta_{\mathrm{io}}+\delta_{\mathrm{jo}}\right)$

(8)

The term ' $\mathrm{o}$ ' denotes the pre-transient conditions. We know that due to sudden load changes, the fault occurs in electrical network and we use the corresponding values in above equations.

\section{SIMULATION MODELLING}

The Simulink model of complete 3-machine and 9 bus system shown in Fig. 3.

The mathematical modeling equation gives the transfer function of the different blocks. The Fig. 3 shows the complete system representation for transient stability. The electrical power outputs for different generators are shown by subsystems 1,2 and 3 as shown in Fig 4, $5 \& 6$. The Simulink model can be run by MATLAB/SIMULINK command line from an $\mathrm{m}$-file program. Similarly, with the help of m-file program all parameters like fault clearing time, initial values etc. due to fault can be controlled. This arrangement of the model is simple and systematic, which is suitable for researchers or students to build the required network and to investigate the effect of the different fault location and different fault conditions i.e. different fault impedance. 


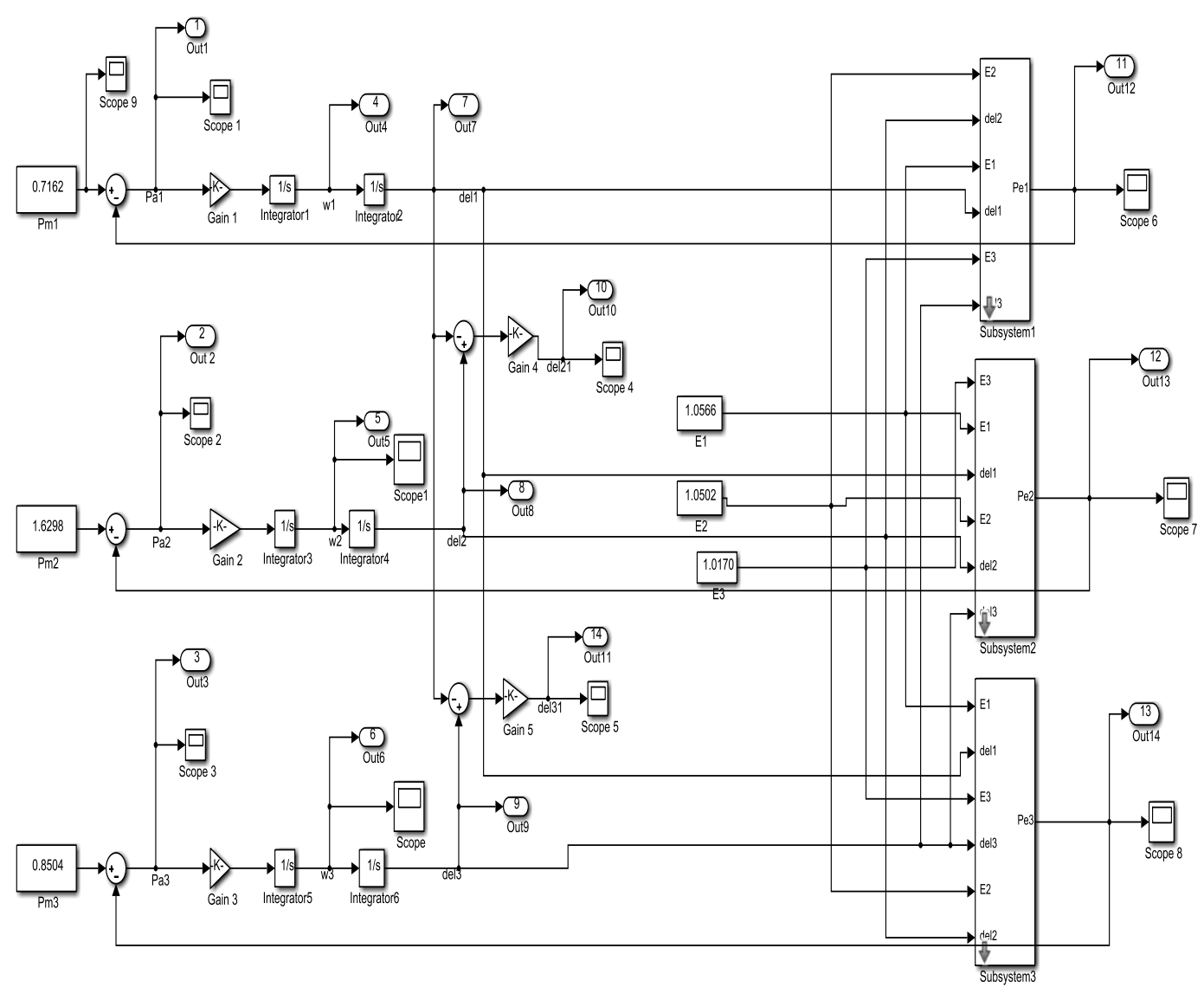

Fig. 3: Complete Simulink model for transient stability analysis of three machines

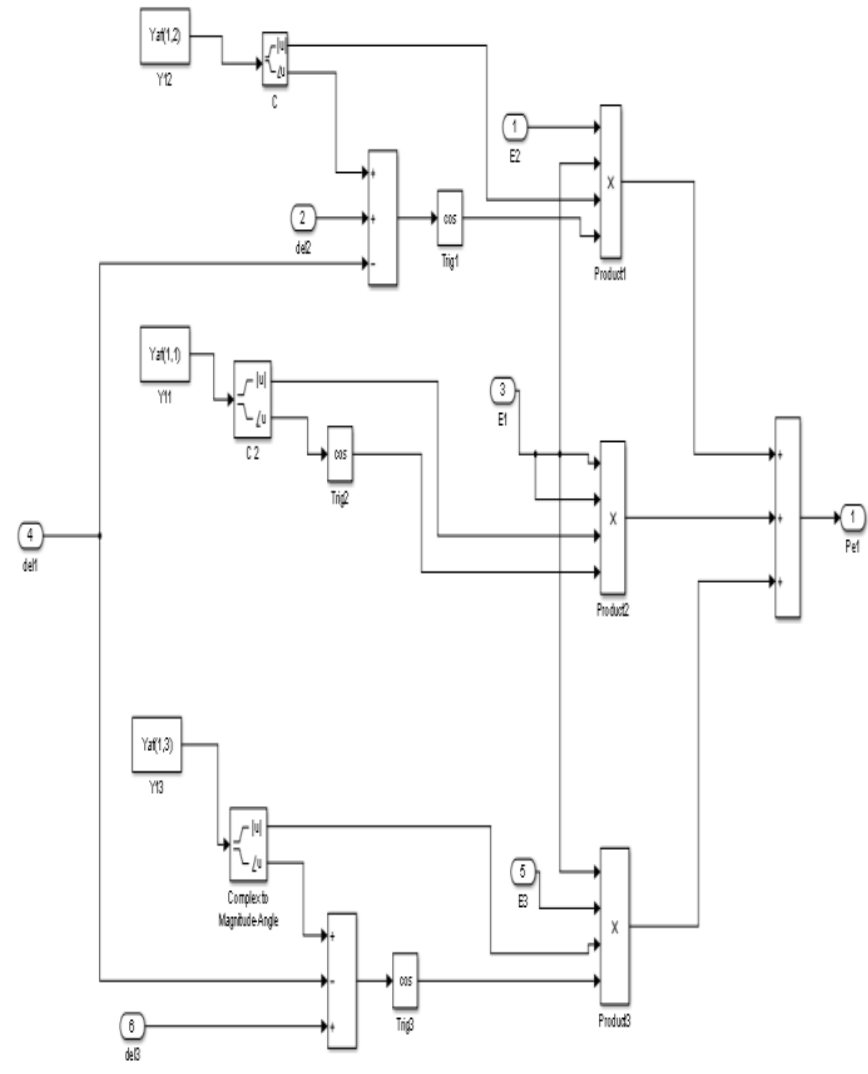

Fig. 4: Computation of electrical power output of generator by subsystem 1

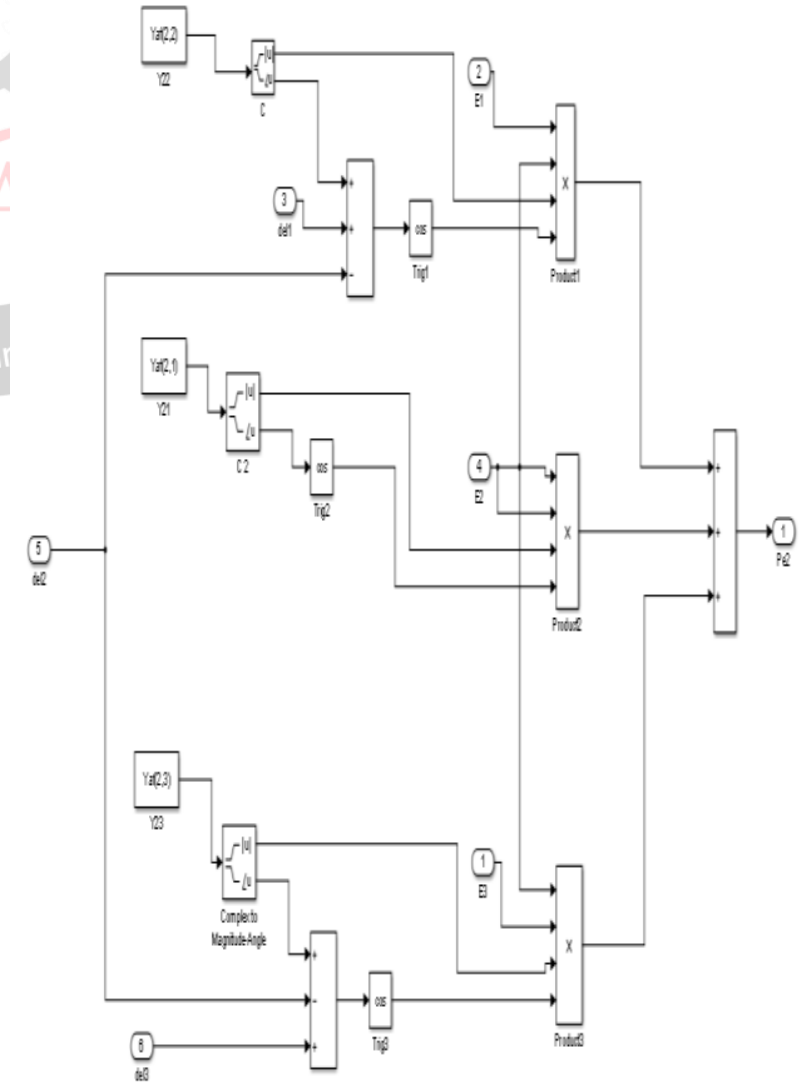

Fig. 5: Computation of electrical power output of generator by subsystem 2 


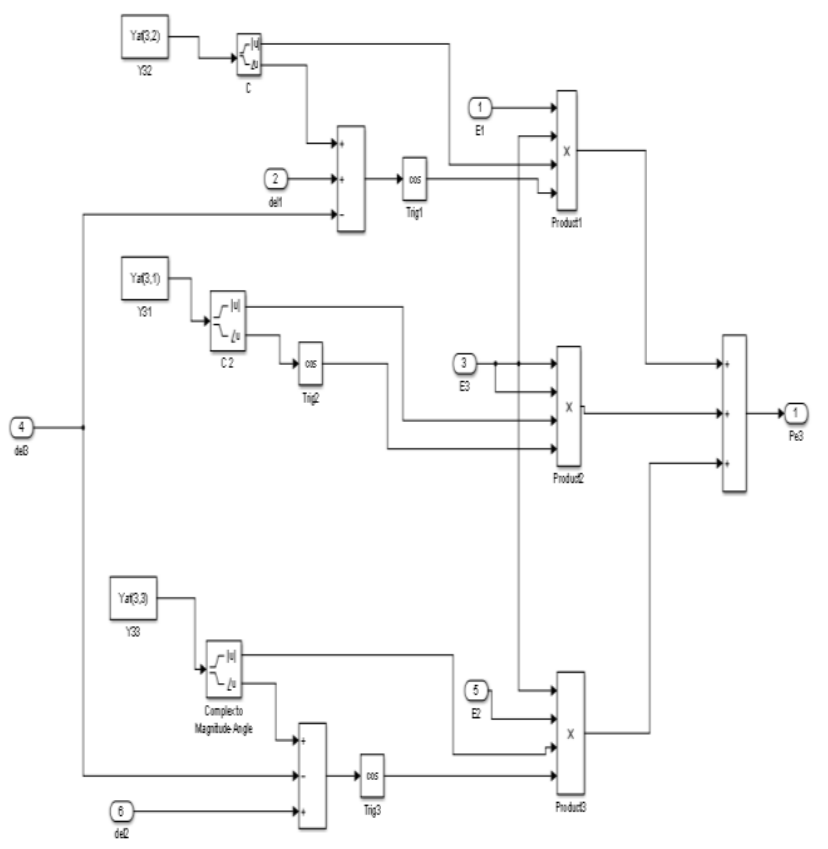

Fig. 6: Computation of electrical power output of generator by subsystem 3

In the Fig. 4, Fig. 5 and Fig. 6 the subsystems for the formulation of electrical power output of all three generators are shown. We use the mathematical modeling equation in the form of Simulink block to solve electrical power output of Generator 1, Generator 2 and Generator 3. Modern power systems have many interconnected generating stations, each with several generators and many loads. So we have presented multi-machine system and its stability has been analyzed using illustration and various fault and provided solution to the fault too.

Transient stability study is based on the application of a three-phase fault. When the fault is cleared, which may involve the removal of the faulty line, the bus admittance matrix is recomputed to reflect the change in the networks.

\section{METHODOLOGY OF MULTIMACHINE SYSTEM}

Assumption in Multi machine stability analysis:

1. All Asynchronous power is ignored.

2. Each synchronous machine is represented by a constant voltage source.

3. The governor's controller action is neglected and the input powers are assumed to remain same (not change).

4. All loads are converted to equivalent admittances to ground using the pre-fault bus data and are assumed to be remain constant.

5. The mechanical rotor angle of each machine coincides with the angle of the voltage behind the machine reactance.
6. Machine belonging to same station swing together and are said to be coherent. A group of coherent machine is represented by one equivalent machine.

Before stability analysis certain preliminary computations are to be done:

1. All system data are calculated on common base-100 MVA.

2. All loads are converted as constant equivalent impedances.

3. Voltage behind the transient reactance is calculated.

The initiation and removal of fault by electrical three phase circuit breakers in a power system exhibit that the system is going through a fault with change in the system configuration in three stages: pre-fault, fault and post-fault stages. The dynamics of the power system during fault and post-fault conditions are nonlinear and the exact solution is very hilarious. In transient stability analysis, particularly, those involving very short periods of analysis, a synchronous machine can be represented by a voltage source behind transient reactance that is same (not change) in magnitude but its angular position has changed.

This representation neglects the effects of saliency and assumes flux linkage never changed at this condition and there is a small change in speed. The voltage behind the transient reactance is determined from the following equation

$$
E_{i}=V_{i}+j X_{d} I_{i}
$$

The Swing Equation is given by:

$$
\mathrm{M} \frac{\mathrm{d}^{2} \theta}{\mathrm{dt}^{2}}=\mathrm{P}_{\mathrm{a}}=\mathrm{P}_{\mathrm{m}}-\mathrm{P}_{\mathrm{e}}
$$

The Critical Clearing Angle is given by:

$$
\mathrm{t}_{\mathrm{cr}}=\left(\frac{2 \mathrm{H}\left(\delta_{\mathrm{cr}}-\delta_{0}\right)}{\pi \mathrm{fP}}\right)^{\frac{1}{2}}
$$

All symbols given in equation (9), (10) \& (11) have their usual meanings.

\section{MATLAB/SIMULINK RESULTS}

The different kind of results we got for different values of fault clearing time (FCT). Fig. 7 shows the individual generator angles and the accelerating power. For the system with FCT $=0.1$ sec., the Fig. 8,9 and 10 shows the rotor angular speed deviations and accelerating powers for the same case. The results show that the power system is stable in this case. We can see in the complete model of Fig. 3 that output ports 7,8 and 9 give the individual generator angles of the respective machines. Ports 10 and 11 (or alternatively Scopes 4 and 5) give the relative angular positions of generators 2 and 3 respectively, with generator 1 as reference. Similarly, ports 4,5 and 6 give the angular velocities of the machines, whereas Scopes 1-3 (or the corresponding ports) display the accelerating powers. 


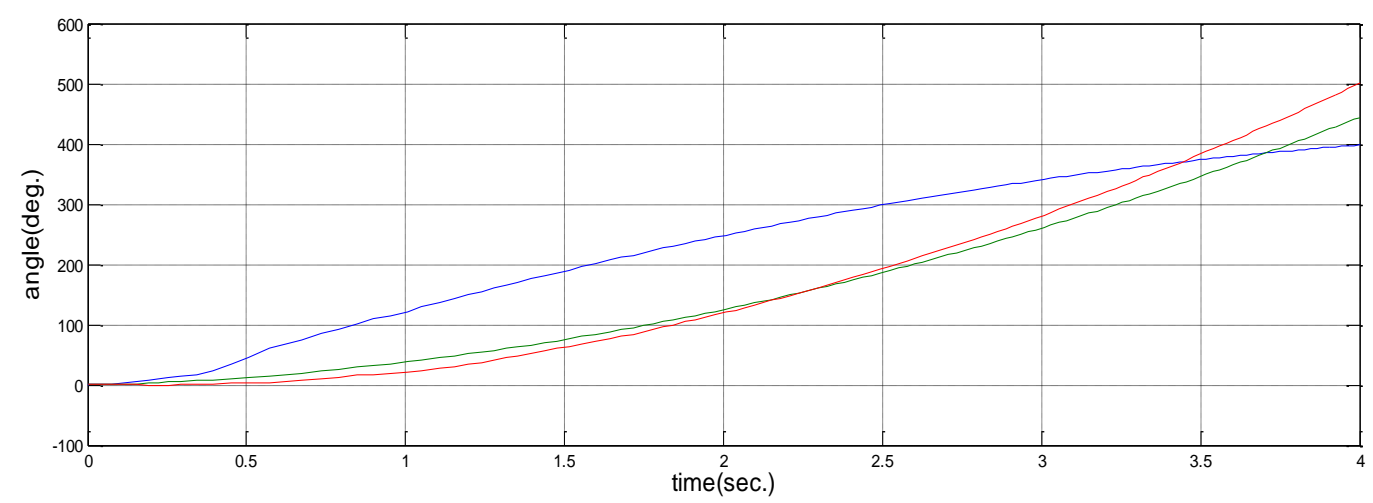

Fig. 7: Angular position of individual generator

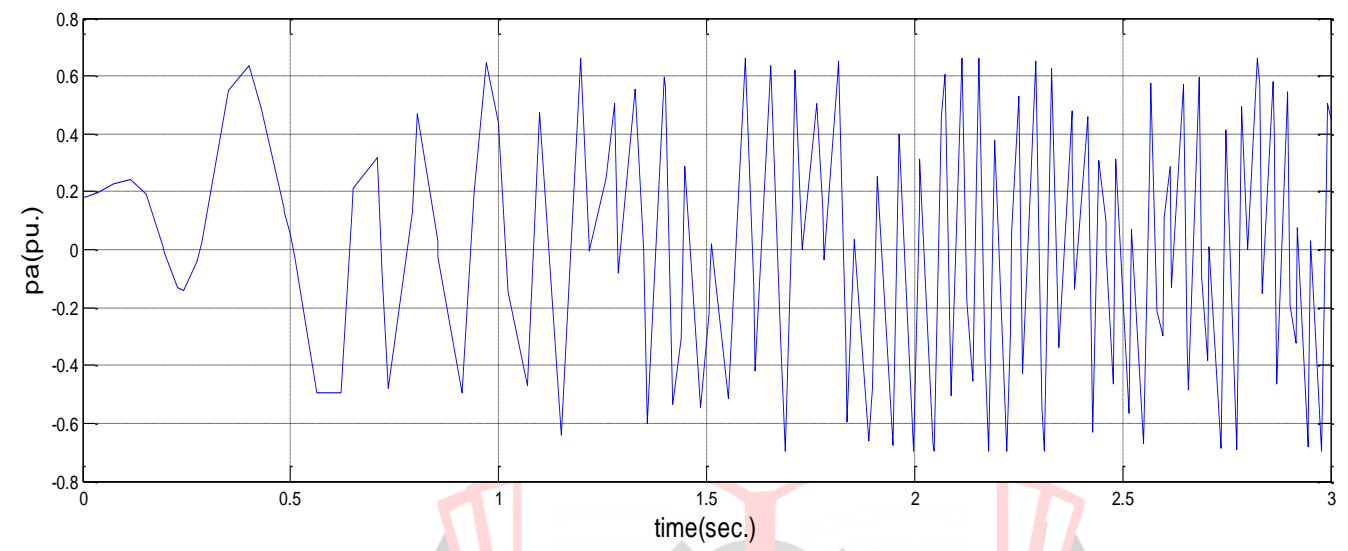

Fig. 8: Plot of generator 1 accelerating power

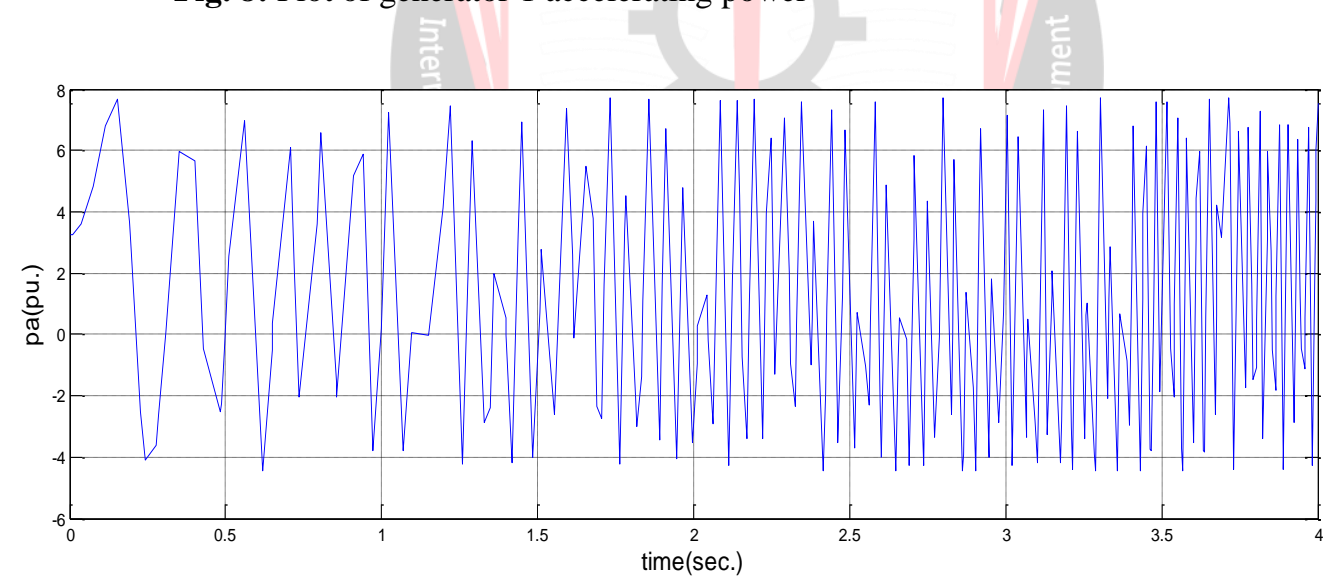

Fig. 9: Plot of generator 2 accelerating power

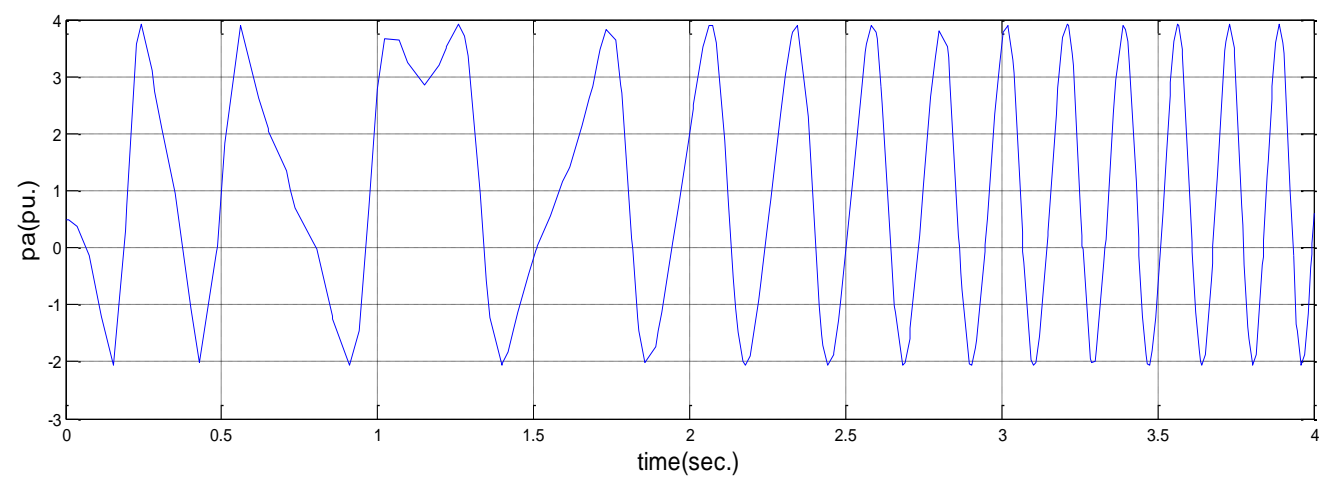

Fig. 10: Plot of generator 3 accelerating power 


\section{CONCLUSION}

The load controlling is very complex in nature day by day. Therefore, we need to concentrate on generation side. The synchronism of the system is maintained on generation side whether the fault is caused by current or voltage. It is advantageous to maintain the efficiency of system as well as for better option for consumer. The overall model for transient stability of a multi-machine power system was developed using MATLAB/Simulation. The angular position of individual generators have been changed during fault and fault is cleared after fault clearing time of $0.1 \mathrm{sec}$ as shown in Fig. 7. Similarly, the accelerating power of all generators varies as shown in Fig. 8, Fig. 9 \& Fig. 10. So, finally we can state that the load angle goes on increasing due to the inertia of the rotor. Now, the output power is greater than the input power and the rotor starts retarding. The system will be stable if the retardation area is equal to the acceleration area depends upon the clearing angle. When clearing angle increases then area also increases, and to find equal areas, load angle is increased till it has a maximum value. This is the maximum allowable value of load angle for stability. For the system to be stable, the clearing angle should be less than the critical clearing angle. If the actual clearing angle is greater than the critical clearing angle, the system become unstable. Thus, more rapidly the fault is cleared, the smaller will the accelerating area, and the greater the chance of stable operation being restored. All this can be done through MATLAB Simulation. The Simulation is nothing but just to represent the system equations in the form of block diagram which is called as Simulation model. The different kinds of blocks are available in the various Simulink libraries as well as toll boxes are available such as Power System Block set, Controls Toolbox, Neural Networks Block set etc. The Simulink model includes the Adding a set of equations (block methods) to each block that define the time-based relationships between the input signals, output signals, and the block state variables. Adding parameters to each block that specify the coefficients from the model equations. Providing a Simulink engine for numerical solution of the block diagram by evaluating the relationships over time, where time starts at a user specified "start time" and ends at a user specified "stop time." Generally, for a transient stability study the model facilitates fast and precise solution of nonlinear differential equations viz. the swing equation. The user can easily select or modify the solver type, step sizes, tolerance, simulation period, output options etc. with the help of an appropriate menu from within Simulink. Any parameter within any block or subsystem of the model can be easily modified through simple MATLAB commands to suit the changes in the original power system network due to fault or a corrective action.

\section{REFERENCES}

[1] P.K Iyambo and R. Tzoneva “ Transient stability Analysis of IEEE 14-Bus Power system” 2007

[2] Huynh Chau Duy,Huynh Quang Minh , "Transient Stability Analysis Of Multimachine System”2003

[3] La Van Ut, "Analysis and Control Stability for Power System" Science and Technology press, 2000.

[4] Prabha Kundur, Power System Stability and Control, Tata McGraw-Hill, Inc 1994.

[5] W.D Stevenson JR. Power System Analysis Tata McGraw Hill Edition

[6] Glen W Stagg Computer methods in Power System Tata McGraw Hill Edition

[7] H.W. Dommel \& W.F. Tinney, “ Optimal power flow solution", IEEE Trans.(Power App.\& Syst.),Vol87,October, 1968.

[8] Divya Prakash1, Er. Vinay Kumar Tripathi PG Student [PS], "Enhancing Stability ofMulti-Machine IEEE 9 Bus Power System Network", "International Journal of Advanced Research in Electrical, Electronics and Instrumentation Engineering", Vol. 4, Issue 5, May 2015

[9] Tefera Mekonnen, Dr Hadadi Sudheendra, Getnet_Zewde "A Unified Approach for the Transient Stability Analysis for a Multi machine System Using MATLAB/ Simulink Model" International Journal of Innovative Research in Engineering \& Multidisciplinary Physical Sciences (IJIRMPS) Volume 3, Issue 3, October 2015.

[10] Simulink User's Guide (The Mathworks, Natick, MA, 1999).

[11] Hadi Saadat, Power System Analysis (McGraw-Hill, New York, 1999). 